\title{
Structural insight into the catalytic mechanism of arsenate reductase from Synechocystis sp. PCC 6803
}

\author{
Y. Yan, J. Ye, X. Zhang, X.M. Xue \& Y.G. Zhu \\ Key Lab of Urban Environment and Health, Institute of Urban Environment, Chinese Academy of Sciences, \\ Xiamen, China
}

\begin{abstract}
Arsenate reductases are the key enzymes in biological arsenic detoxification by catalyzing the intracellular reduction of arsenate to arsenite, and arsenite could be subsequently pumped out of the cells by ArsB or Acr3. The arsenate reductase from cyanobacterium Synechocystis sp. Strain PCC 6803 (SynArsC) shows sequence homology with the thioredoxin-dependent arsenate reductase family, while utilizes the glutathione/glutaredoxin system for arsenate reduction. SynArsC is classified as a novel thioredoxin/glutaredoxin hybrid arsenate reductase family. Here we report the crystal structures of SynArsC in the native and phosphatebound states at 1.37 and $1.55 \AA$ resolutions, respectively. The structures are mostly similar, but also show differences with implications in SynArsC's arsenate reduction mechanism. Our results provide insights into SynArsC's structure-function relationship and its enzymatic mechanism.
\end{abstract}

\section{INTRODUCTION}

Arsenic is a toxic metalloid element that causes numerous environment and health problems. Ubiquitous arsenic forced living organisms to evolve different mechanisms for arsenic resistance. A two-step process, arsenate reduction followed by arsenite efflux, is a well-known mechanism of arsenate resistance (Zhu et al., 2017).

Cytoplasmic arsenate reductases (ArsC) are divided into at least three families (Messens \& Silver, 2006). The first family is represented by R773ArsC from Escherichia coli, which uses glutathione (GSH)/glutaredoxin (Grx) as electron donor and has a single catalytic cysteine. The second family is exemplified by SaArsC from Staphylococcus aureus, which uses thioredoxin $(\operatorname{Trx})$ as a reducing system and requires three cysteines for arsenate reduction. The last family is termed as Acr2P, and only present in eukaryotic organisms, such as Saccharomyces cerevisiae and Arabidopsis thaliana.

Arsenate reductase from Synechocystis $s p$. PCC 6803 (SynArsC) is a novel ArsC belonging to the Trx/Grx hybrid arsenate reductase family. The primary sequence of SynArsC is similar with that of Trxdependent ArsCs, whereas SynArsC utilized Grx/GSH system for arsenate reduction (López-Maury et al., 2009; Li et al., 2003). Cys8, Cys80 and Cys 82 in SynArsC were identified as essential cysteine residues by site-directed mutagenesis, and the Cys $80 / \mathrm{Cys} 82$ disulfide was detected by equilibrium redox titrations. In order to elucidate the special mechanism of $\mathrm{As}(\mathrm{V})$ reduction, we determined the crystal structures of native SynArsC and its complex with phosphate $\left(\mathrm{PO}_{4}^{3-}\right)$.

\section{METHODS/EXPERIMENTAL}

\subsection{Purification and crystallization}

SynArsC with his-tag was overexpressed in E. coli Rosetta (DE3), and purified by Ni-NTA agarose column and size-exclusion chromatography. Crystallization trials were set up at $291 \mathrm{~K}$ using hangingdrop vapor-diffusion method. The $2 \mu \mathrm{L}$ hanging drops consisted of $1 \mu \mathrm{L}$ protein solution and $1 \mu \mathrm{L}$ reservoir solution were equilibrated against $500 \mu \mathrm{L}$ reservoir solution $(32 \%(w / v)$ PEG 3350, $100 \mathrm{~m} M$ citric acid, $\mathrm{pH}$ 5.5). Needle-like crystals were observed after one week. As the co-crystallization of SynArsC and $\mathrm{As}(\mathrm{V}) / \mathrm{As}(\mathrm{III})$ was unsuccessful, we crystallized SynArsC with $\mathrm{PO}_{4}^{3-}$, an analog of $\mathrm{As}(\mathrm{V})$. The crystals of SynArsC- $\mathrm{PO}_{4}^{3-}$ were obtained in optimized reservoir solution consisting of $28 \%(w / v)$ PEG 3350, $100 \mathrm{~m} M$ citric acid and $\mathrm{pH} 4.8$ by adding $5 \mathrm{mM}$ $\mathrm{NaH}_{2} \mathrm{PO}_{4}$ into the protein solution. Crystals were mounted and soaked in corresponding reservoir solution supplemented with $20 \%$ glycerol and flash-cooled in liquid nitrogen prior to data collection.

\subsection{Data collection, structure determination and refinement}

Diffraction data were collected with a wavelength of $0.9792 \AA$ at $100 \mathrm{~K}$ on beamline BL17U of Shanghai Synchrotron Radiation Facility (SSRF). All data were indexed and integrated with iMosflm, and scaled with AIMLESS from CCP 4 program suite. The structures of native SynArsC and SynArsC-PO ${ }_{4}^{3-}$ were obtained by the molecular replacement method using Phaser. A single structure modified from the 20 conformers 
of NMR structure of SynArsC (Yu et al., 2011) was used as a search model. The structure models were built using Coot, and refined by iterative rounds of PHENIX automatically and Coot manually.

\section{RESULTS AND DISCUSSION}

\subsection{Determination of SynArsC structures}

The crystal structure of native SynArsC was determined in space group $P 22_{1} 2_{1} 2_{1}$, with unit-cell parameters $a=33.03, b=33.35, c=107.57 \AA$. The structure of SynArsC- $\mathrm{PO}_{4}^{3-}$ belonged to space group $C 121$, with unit-cell parameters $a=64.26, b=33.37$, $c=56.63 \AA, \beta=100.2^{\circ}$. The native SynArsC and SynArsC- $\mathrm{PO}_{4}^{3-}$ structures were refined to resolutions of $1.37 \AA$ and $1.55 \AA$, respectively. The native SynArsC was modelled and refined with a final $R_{\text {work }}$ of 0.166 and an $R_{\text {free }}$ of 0.194 . The final $R_{\text {work }}$ and $R_{\text {free }}$ for SynArsC-PO ${ }_{4}^{3-}$ structure were 0.152 and 0.184 , respectively.

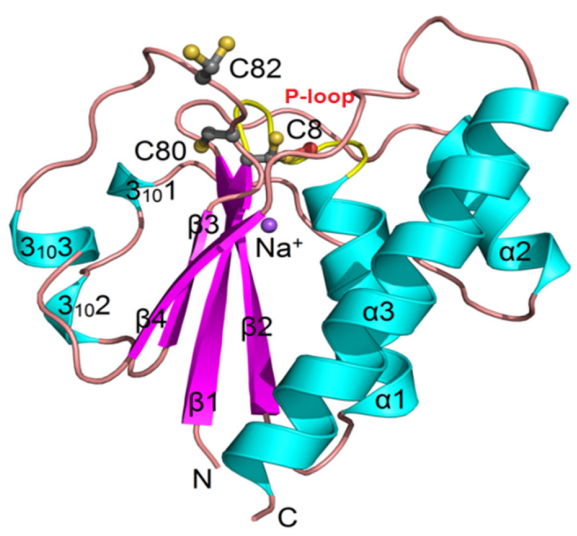

Figure 1. The overall structure of SynArsC shown as cartoon representations. Key residues are shown in stick representation. The P-loop is colored yellow.

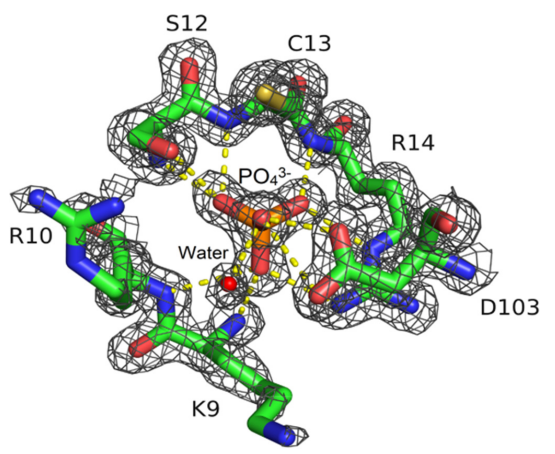

Figure 2. The binding site in the SynArsC- $\mathrm{PO}_{4}^{3-}$ structure. The $2 F_{o b s}-F_{\text {calc }}$ density map was calculated from the refined model contoured at $1.0 \sigma$.

\subsection{Overall structure of SynArsC}

SynArsC showed similar structure with Trx-dependent ArsCs, consisting of a four-stranded and parallel opentwisted $\beta$-sheet $(\beta 1-4)$ flanked by three major $\alpha$ helices $(\alpha 1-3)$ and three small $3_{10}$-helices $\left(3_{10} 1-3\right)$ on both sides (Fig. 1).

In the structure of SynArsC, the conserved $\mathrm{P}$-loop connecting the first $\beta$-sheet with $\alpha$-helix is an active site of both phosphatases and arsenate reductases. The first conserved cysteine (cys8) was in the P-loop. However, the other active residues were in unique geometries, with the last two conserved cysteines (cys80 and cys82) locating in the flexible loop and the conserved aspartic acid (Asp103) being in the rigid area.

\subsection{The phosphate-binding site}

$\mathrm{PO}_{4}^{3-}$ is tightly hydrogen-bound to the P-loop in the SynArsC- $\mathrm{PO}_{4}^{3-}$ structure (Fig. 2). The P-loops are almost in the same geometry with Asn11 in the $\alpha \mathrm{L}$ conformation with or without binding to $\mathrm{PO}_{4}^{3-}$. Therefore, the biding of $\mathrm{PO}_{4}^{3-}$ does not remarkably change the conformation of SynArsC. However, it does make the structure more stable. The detailed snapshot also provides insight into the catalytic mechanism of SynArsC.

\section{CONCLUSIONS}

SynArsC showed similar structure with Trx-coupled ArsCs, but also has its own particularity in structure as a member of new arsenate reductase family.

\section{ACKNOWLEDGEMENTS}

This work is supported by the National Natural Science Foundation of China (21507125 and 31270161).

\section{REFERENCES}

López-Maury, L., Sánchez-Riego, A.M., Reyes, J.C. \& Florencio, F.J. 2009. The glutathione/glutaredoxin system is essential for arsenate reduction in Synechocystis sp. strain PCC 6803. J. Bacteriol. 191: 3534-3543.

Li, R., Haile, J.D. \& Kennelly, P.J. 2003. An arsenate reductase from Synechocystis sp. strain PCC 6803 exhibits a novel combination of catalytic characteristics. J. Bacteriol. 185: 6780-6789.

Messens, J. \& Silver, S. 2006. Arsenate reduction: thiol cascade chemistry with convergent evolution. J. Mol. Biol. 362: 1-17.

Yu, C., Xia, B. \& Jin, C. 2011. ${ }^{1} \mathrm{H},{ }^{13} \mathrm{C}$ and ${ }^{15} \mathrm{~N}$ resonance assignments of the arsenate reductase from Synechocystis sp. strain PCC 6803. Biomol. NMR Assignm. 5: 85-87.

Zhu, Y.G., Xue, X.M., Kappler, A., Rosen, B.P. \& Meharg A.A. 2017. Linking genes to microbial biogeochemical cycling: lessons from arsenic. Environ. Sci. Technol. 51(13): 7326-7339. 